\title{
Key Players in Choline Metabolic Reprograming in Triple-Negative Breast Cancer
}

\author{
Egidio lorio ${ }^{1 *}$, Maria José Caramujo ${ }^{1 \dagger}$, Serena Cecchetti ${ }^{1}$, Francesca Spadaro ${ }^{2}$, \\ Giulia Carpinelli', Rossella Canese ${ }^{1}$ and Franca Podo ${ }^{1}$
}

${ }^{1}$ Department of Cell Biology and Neurosciences, Istituto Superiore di Sanità, Rome, Italy, ${ }^{2}$ Department of Hematology, Oncology and Molecular Medicine, Istituto Superiore di Sanità, Rome, Italy

OPEN ACCESS

Edited by:

Kristine Glunde,

Johns Hopkins School of Medicine,

USA

Reviewed by:

Hadassa Degani,

Weizmann Institute of Science, Israel

Balaji Krishnamachary,

Johns Hopkins University, USA

Jerry David Glickson,

University of Pennsylvania, USA

*Correspondence: Egidio lorio

egidio.iorio@iss.it

'Present address:

Maria José Caramujo,

Faculdade de Ciências,

Centre for Ecology, Evolution

and Environmental Changes (CE3C), Universidade de Lisboa, Lisboa, Portugal

Specialty section: This article was submitted to Cancer Imaging and

Diagnosis, a section of the journal Frontiers in Oncology

Received: 02 April 2016 Accepted: 12 September 2016 Published: 30 September 2016

Citation:

Iorio E, Caramujo MJ, Cecchetti S, Spadaro F, Carpinelli G, Canese $R$ and Podo F (2016) Key Players in Choline Metabolic Reprograming in

Triple-Negative Breast Cancer.

Front. Oncol. 6:205.

doi: 10.3389/fonc.2016.00205
Triple-negative breast cancer (TNBC), defined as lack of estrogen and progesterone receptors in the absence of protein overexpression/gene amplification of human epidermal growth factor receptor 2, is still a clinical challenge despite progress in breast cancer care. ${ }^{1} \mathrm{H}$ magnetic resonance spectroscopy allows identification and non-invasive monitoring of TNBC metabolic aberrations and elucidation of some key mechanisms underlying tumor progression. Thus, it has the potential to improve in vivo diagnosis and follow-up and also to identify new targets for treatment. Several studies have shown an altered phosphatidylcholine (PtdCho) metabolism in TNBCs, both in patients and in experimental models. Upregulation of choline kinase-alpha, an enzyme of the Kennedy pathway that phosphorylates free choline (Cho) to phosphocholine (PCho), is a major contributor to the increased PCho content detected in TNBCs. Phospholipase-mediated PtdCho headgroup hydrolysis also contributes to the build-up of a PCho pool in TNBC cells. The oncogene-driven PtdCho cycle appears to be fine tuned in TNBC cells in at least three ways: by modulating the choline import, by regulating the activity or expression of specific metabolic enzymes, and by contributing to the rewiring of the entire metabolic network. Thus, only by thoroughly dissecting these mechanisms, it will be possible to effectively translate this basic knowledge into further development and implementation of Cho-based imaging techniques and novel classes of therapeutics.

Keywords: triple-negative breast cancer, phosphatidylcholine metabolism, metabolic reprograming, phospholipase, choline kinase

\section{INTRODUCTION}

Classification of breast cancer (BC) has been historically based on both analysis of tumor morphology and histological detection of three marker proteins: the estrogen receptor (ER), the progesterone receptor (PR), and the human epidermal growth factor (EGF) receptor tyrosine kinase 2 (ErbB2 or HER2). Tumors which express none of these three markers are collectively referred to as triplenegative breast cancer (TNBC; $\mathrm{ER}^{-}, \mathrm{PR}^{-}, \mathrm{HER} 2^{-}$) and still pose a clinical challenge. More recently, gene expression analyses showed that $\mathrm{BC}$ is a more heterogeneous disease than previously assumed and the $\mathrm{BC}$ histotypes based on ER/PR/HER2 classification were expanded to include five major transcriptional subtypes: basal-like, HER2-enriched, luminal A, luminal B, and normal breastlike $(1,2)$. The majority $(70-80 \%)$ of TNBCs are defined as basal-like by gene expression (3) and 
share other molecular features with this BC subtype. According to most epidemiological studies, TNBCs represent $10-20 \%$ of all BCs, although a higher proportion can be found in some ethnic groups and among BRCA1 mutation carriers. TNBCs are typically poorly differentiated, frequently have high histological grade and mitotic index, and often present early onset and shorter disease-free and overall survival $(4,5)$. Recent large-scale gene expression and genome-based studies have shown that TNBC is a heterogeneous disease (Figure 1A) comprising at least four to six definable molecular subtypes that express elements of distinct oncogenic signaling pathways $(6,7)$. Interestingly, $50-70 \%$ of all TNBCs overexpress the EGF receptor tyrosine kinase 1 (ErbB1, EGFR, or HER1). EGFR is one of the major regulators of survival, proliferation, and migration (8), leading to activation of the phosphoinositide 3-kinase (PI3K) and ERK pathways, which in turn stimulate other receptor-activated signaling cascades associated with cancer onset and progression (Figure 1B) (9).

The aggressiveness of TNBC and the lack of targeted therapies specifically recommended for TNBC patients highlight the need to explore additional molecular mechanisms beyond genomic and proteomic changes to better elucidate the metabolic pathways required for TNBC growth and survival $(10,11)$.

\section{METABOLIC REPROGRAMING IN TNBC CELLS}

Molecular genomic and proteomic studies have been carried out to understand the complexity of TNBC and identify markers that can be therapeutically targeted. However, little is known about the metabolic alterations that distinguish TNBC from non-triple-negative subtypes and characterize TNBC progression. Previous studies have shown that proteins that are involved in glycolysis, glutaminolysis, and glycine or serine metabolism are differentially expressed among different BC subtypes in tissue microarray sections and in a large series of invasive $\mathrm{BC}$ specimens (12-14). In particular, several observations reported that TNBC have elevated glucose uptake and a glycolytic gene/ protein expression signature $(12,14)$. A recent study reported a novel mechanism, whereby the transcription factor $\mathrm{c}-\mathrm{Myc}$ drives glucose metabolism in TNBC MDA-MB-157 cells by direct repression of thioredoxin-interacting protein (TXNIP), a potent negative regulator of glucose uptake, aerobic glycolysis, and glycolytic gene expression. A Myc $\mathrm{c}^{\text {high}} / \mathrm{TXNIP}^{\text {low }}$ gene signature correlates with decreased overall patient survival and decreased metastasis-free survival in BC. The correlation between the $\mathrm{Myc}^{\text {high }} / \mathrm{TXNIP}^{\text {low }}$ gene signature and poor clinical outcome is evident only in TNBC, not in other BC subclasses. Furthermore, mutation in p53 (TP53), found in the majority of TNBCs, enhances the correlation between the $\mathrm{Myc}^{\text {high }} / \mathrm{TXNIP}^{\text {low }}$ gene signature and death from BC (15). Finally, an increase of glycolysis was found in a panel of five TNBC cells and accumulation of fructose-1,6-bisphosphate (F1,6BP), a glycolytic intermediate that directly binds to and enhances the activity of EGFR, was detected in MDA-MB-468 cells, with enhanced lactate excretion, tumor growth, and immune escape (16). TNBC cell lines, such as MDA-MB-468, MDA-MB-231, MDA-MB-436, and BT20, exhibit function defects in multiple respiratory complexes with a reduction in expression of complex I and complex III proteins of the mitochondrial respiratory chain relative to receptor-positive cell lines (17). The increased glycolytic activity in TNBCs could be responsible for the increased ${ }^{18} \mathrm{~F}$-fluorodeoxyglucose (FDG) uptake generally reported in PET examinations of these patients, beyond a large variability in the maximum and mean standardized uptake values and in metabolic volumes (18-21).

Lipid metabolism activation in BC cells is recognized as a hallmark of carcinogenesis $(22,23)$. Increased fatty acid (FA) synthesis due to increased levels of fatty acid synthase (FAS) has been observed in various cancers and is correlated with a poor prognosis in many instances (23). FAS could, in principle, be an appealing therapeutic target because most cancer cells depend on FAS-mediated de novo FA synthesis, whereas most healthy cells prefer to incorporate exogenous FAs (24). However, reports on the overexpression of FAS across BC subtypes, and in TNBC in particular, are still contradictory (25-27), and further studies are needed before considering this enzyme as a strong therapeutic target.

The concerted activation of an assembly of molecular complexes in cancer cells cooperates to sustain an oncogene-induced cell signaling through multiple postreceptor pathways involved in phospholipid biosynthesis and breakdown. Among these, phosphatidylinositol 4-phosphate 5-kinase Igamma (PIPKI $\gamma$ ) is overexpressed in TNBC cells, in which the loss of this enzyme impairs PI3K/Akt activation (28). Furthermore, two major enzymes involved in the agonist-induced phosphatidylcholine (PtdCho) cycle, such as choline kinase (ChoK) and PtdChospecific phospholipase C (PC-PLC), are overexpressed and activated in various BC subtypes, including TNBC cells, with the implications on expression and oncogenic function of EGF receptors' family members (29-33).

The present evidence points to the existence of multiple links between enzymes involved in the glycolytic gene/protein signature and those responsible for enhanced carbon fluxes through the oncogene-driven PtdCho biosynthesis and catabolism in BC cells (Figure 2). This biochemical interplay may also serve as a key regulator of tumor progression in TNBCs.

\section{PtdCho METABOLISM IN TNBC}

The introduction of magnetic resonance spectroscopy (MRS) in cancer biology allowed the detection of abnormal profiles of aqueous total choline-containing metabolites (tCho) of the PtdCho cycle in cancer cells and tissues, both at preclinical and clinical level $(9,34-37)$. Substantially modified ${ }^{1} \mathrm{H}$ MRS tCho spectral profiles have been reported on malignant transformation of human mammary (38-40) and prostate epithelial cells (41) and in ovarian cancers (42-44). These modifications occur in the 3.20-3.24 ppm ${ }^{1} \mathrm{H}$ MRS spectral region and are typical of the trimethylammonium headgroups of PtdCho precursors and catabolites, such as phosphocholine (PCho), glycerophosphocholine (GPCho), and free choline (Cho).

Phospholipids play the dual role of being basic structural components of membranes and acting as substrates of reactions involved in key regulatory functions in mammalian cells $(45,46)$. 
A

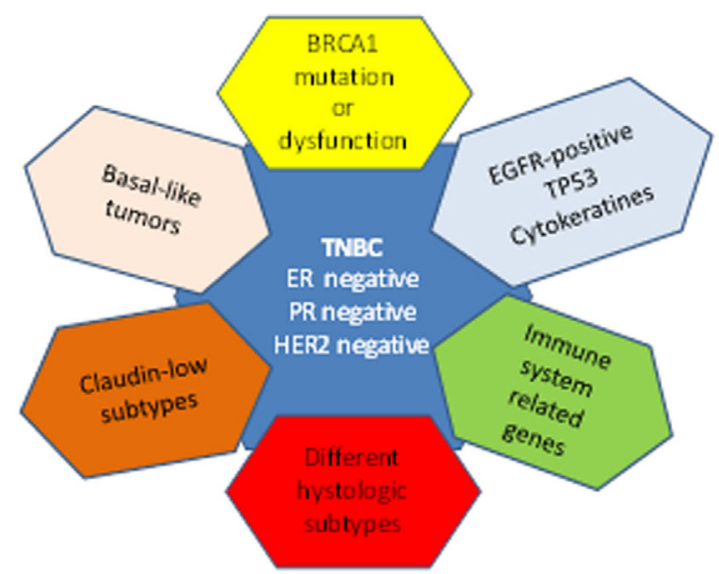

B

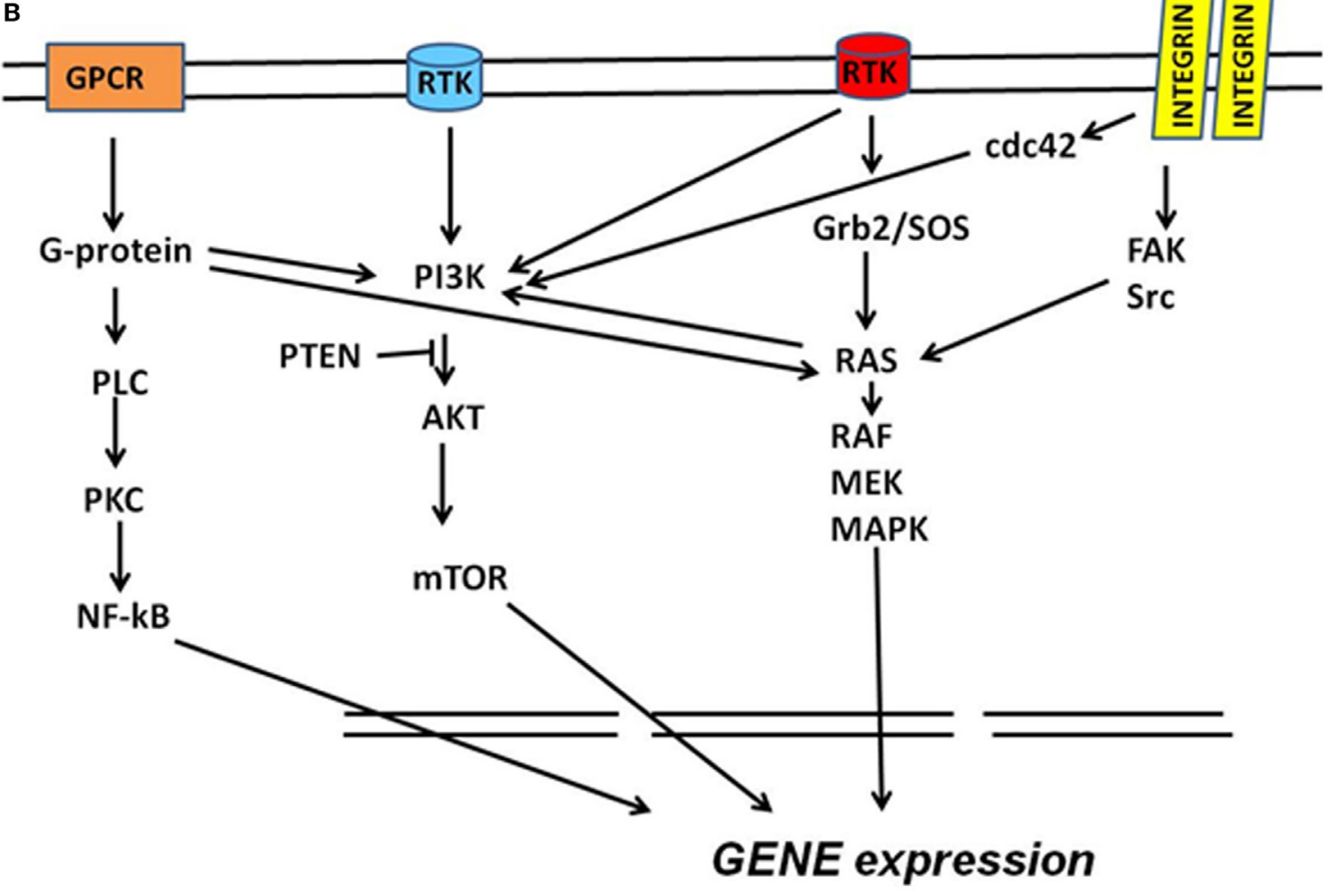

Cell proliferation, survival and invasion

FIGURE 1 | (A) Molecular subclassification of triple-negative breast cancer (TNBC) based on gene expression profiling. Triple-negative breast cancers have been defined as tumors that are devoid of the expression of estrogen receptor (ER), progesterone receptor (PR), and human epidermal growth factor receptor 2 (HER2). TNBC overlap with (1) basal-like breast cancers; (2) BRCA-mutated tumors; (3) claudin-low tumors; (4) tumors overexpressing EGFR, associated with TP53 mutations or expressing cytokeratins; (5) tumors characterized by immune response signatures; and (6) tumors possessing some special histological types. (B) Schematic molecular pathway identified in TNBC. Multiple signaling cascades are activated in TNBC including those triggered by receptor tyrosine kinases (RTK), G protein-coupled receptor (GPCR), and integrins and their downstream effectors. Ras-mediated signaling commonly occurs through the RTK/growth factor receptor-bound protein 2 (Grb2)/Sos-Ras pathway. Ras directly interacts with and activates Raf. Raf phosphorylates and activates MEK, which in turn phosphorylates and activates MAPKs. Integrin engagement triggers several signaling cascades including those that are mediated by FAK, Src, and cdc42. Activation of RTK and other external stimuli lead to the activation of PI3K pathway. PI3K activates AKT (whereas PTEN inhibits this activation) and then mTOR. The G-proteins bind and activate phospholipase $\mathrm{C}$ and activate the nuclear factor kappa B (NF-kB) transcription factor. This network of cell signaling pathways result in the activation of transcription factors that drive genomic signature programs of dysregulated cell cycle progression, proliferation, invasion, and survival.

Hydrolysis of PtdCho, the most abundant phospholipid in eukaryotic cell membranes, can generate second messengers, such as diacylglycerol (DAG), phosphatidic acid (PA), lysophosphatidic acid (LPA), arachidonic acid (AA), and lysophosphatidylcholine (LPtdCho). These PtdCho metabolites are produced through three major catabolic pathways, respectively, mediated by specific 
phospholipases of type C (PC-PLC) and D (PLD), which act at the two distinct phosphodiester bonds of the PtdCho headgroup, and by phospholipases of type A2 and A1 (PLA2 and PLA1), which act in the deacylation reaction cascade (Figure 2). PCho accumulation either produced by ChoK in the first reaction of the three-step Kennedy biosynthetic pathway or by PLC-mediated PtdCho catabolism is associated with tumor growth and progression $(9,33-36)$.
This accumulated evidence supports the inclusion of an altered phospholipid metabolism as a novel candidate hallmark for cancer and as a key regulator in the overall cancer metabolic reprograming. An aberrant PtdCho metabolism associated with increases in the intracellular total choline-containing PtdCho metabolites (tCho) and phosphocholine (PCho) contents (femtomoles per cubic micrometer cell) were initially observed in $\mathrm{BC}$ cells as they progressed from normal to malignant phenotypes,

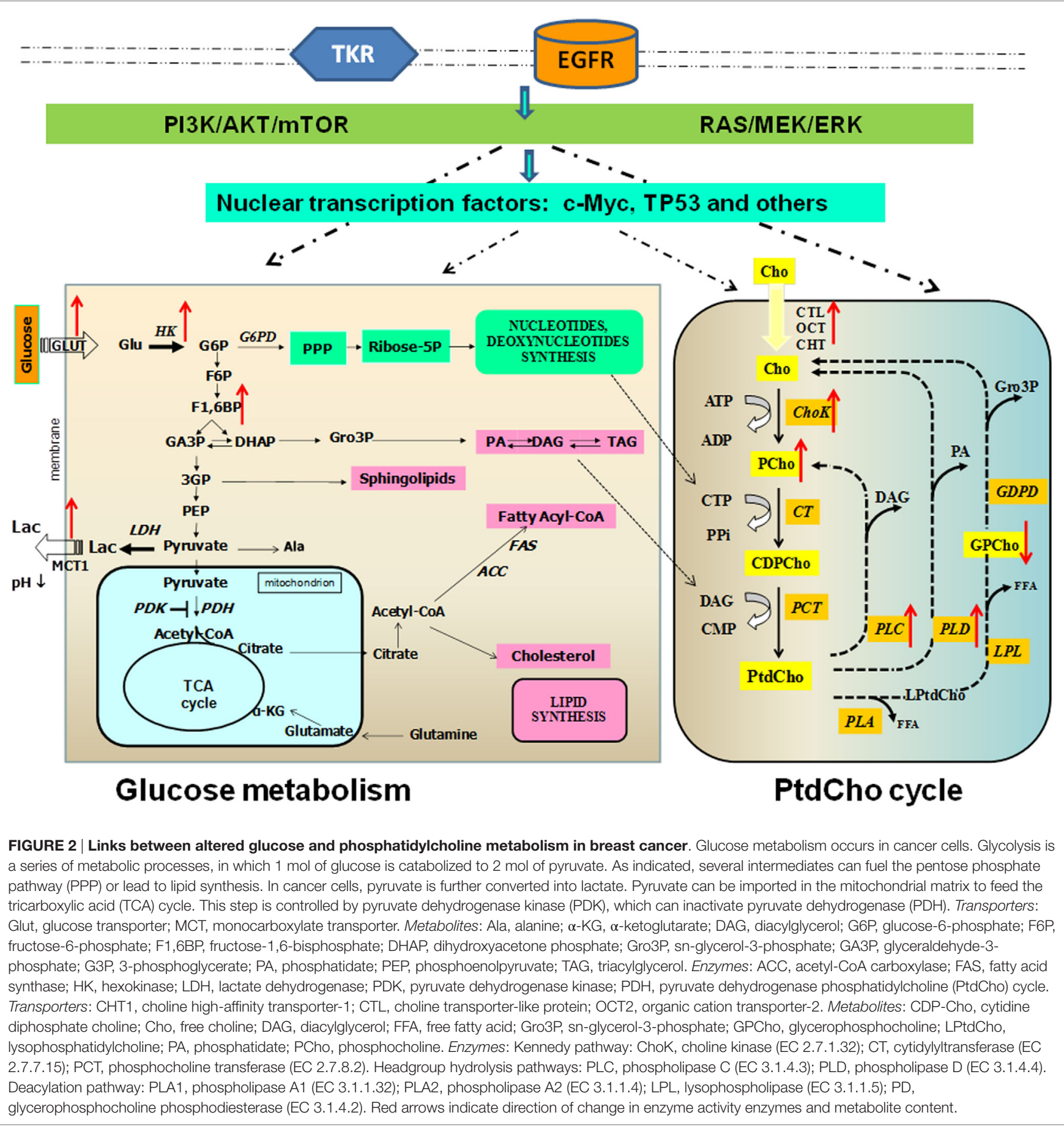


i.e., from non-tumoral immortalized MCF-12A to the highly metastatic TNBC MDA-MB-231 and MDA-MB-435 cell lines (38). The high intracellular content of PCho seemed unrelated to the demand for cell membrane biosynthesis in the investigated BC cells characterized by different doubling time values (38). Notably, a different rate of increase was observed for PCho $(6 \times)$ and PtdCho $(1.5 \times)$ in MDA-MB-231 BC cells compared with the non-malignant MCF12A cells (40). An integration of MRS with gene microarray analysis revealed that a combination of upregulated ChoK and PLD and/or an increased PC-PLC expression/ activity caused PCho accumulation in MDA-MB-231 cells, while lower levels of GPCho were consistent with underexpression of cytosolic calcium-dependent PLA2 group IVA and lysophospholipase 1 (40). Elevated levels of ChoK $\alpha$ and PLD1 isoforms were found in both the $\mathrm{ER}^{-}$MDA-MB-231 BC cell line and in patient-derived $\mathrm{ER}^{-} \mathrm{BC}$ specimens, as compared with the corresponding non-metastatic $\mathrm{ER}^{+} \mathrm{MCF}-7 \mathrm{BC}$ cell line and $\mathrm{ER}^{+}$patient-derived $\mathrm{BC}$ samples (47). Downregulation of ChoK $\alpha$ by RNA silencing increased PLD1 expression, and downregulation of PLD1 increased ChoK $\alpha$ expression, indicating a close relationship between ChoK and PLD enzymes (47). Additionally, ChoK $\alpha$ silencing resulted in increased PC-PLC protein expression (e.g., twofold in MDA-MB-231) suggesting that $\mathrm{BC}$ cells could compensate for the loss of $\mathrm{ChoK} \alpha$ protein levels with PC-PLC upregulation, thus maintaining an intracellular PCho pool size markedly higher than that of non-tumoral breast epithelial cells $(48,49)$.

Although the role of PLAs in BC cells remains unknown, very low expression of secreted PLA2 (sPLA2) was found in basal-like breast tumor biopsies and cultured cells (50). As noted by the authors, the mRNA expression of sPLA2s belonging to IIA, III, and $\mathrm{X}$ groups is regulated by DNA methylation and histone deacetylation, and all three genes are significantly silenced in aggressive TNBC cells due to both mechanisms. It may be interesting to investigate a possible relationship with the regulation of cytosolic PLA2s underexpressed in TNBC apparently linked to low levels of GPCho in this BC subtype (40).

A study by Eliyahu and colleagues (39) confirmed an altered PtdCho metabolism in different molecular BC subtypes relative to human mammary epithelial cells (HMECs), although the MDA-MB-231 TNBC cells exhibited the lowest PCho/NTP ratio among the investigated $\mathrm{BC}$ cell lines. Interestingly, under the adopted experimental conditions, the PCho level in HMEC and $\mathrm{BC}$ cells was found to correlate with Cho transport into the cells, mainly due to the organic cation transporter-2 (OCT2) and the choline high-affinity transporter-1 (CHT1), but not with ChoK activity, suggesting that this step is fast and not rate limiting, although its induction ensures increased PCho levels. The upregulation of choline transporters and ChoK may be related to a cascade of genetic changes that are associated with the multistep process of carcinogenesis (51).

Enzymatic assays showed a twofold to sixfold activation of PC-PLC in BC of different subtypes compared with a non-tumoral counterpart (MCF-10A cell line) (32). The activity rate measured in the TNBC MDA-MB-231 cell line was about twofold higher than that of HER2-enriched and ER-positive cell lines. Metabolic analysis of MDA-MB-231 cells identified a characteristic biochemical signature of these cells relative to the non-tumoral MCF-10A counterpart, consisting of higher contents of PCho and succinate, elevated proportion of monounsaturated FAs and increased ChoK and PC-PLC protein expression (52). The importance of the cell membrane lipid profile to discriminate $\mathrm{BC}$ subtypes is receiving increasing attention, and the results suggest possible links between altered metabolic pathways in $\mathrm{BC}$ and membrane molecular rearrangement. He and colleagues (53) could discriminate BC cell lines from MCF-10A cells on the basis of phospholipid species composition and expression of five lipogenesis-related enzymes. The authors suggested that elevated expression levels of fatty acid synthase 1 (FAS1), stearoyl-CoA desaturases 1 and 5 (SCD1 and SCD5), and ChoK $\alpha$ may be closely related to enhanced levels of saturated and monounsaturated lipids in BC cell lines. Most interestingly, it was possible to distinguish adequately between MDA-MB-231 cells based on the highest level of PtdCho (36:1) and PtdCho (36:2) and ChoKo expression compared with other BC subtypes (53).

Differences in phospholipid and lipid metabolism between cells in culture and in solid tumors have been detected by in vivo MRS in the MDA-MB-231 model. These differences may be ascribed to characteristic conditions of solid tumor microenvironment such as depletion of nutrients and oxygen, changes in $\mathrm{pH}$, and interactions between cancer and stromal/endothelial cells $(54,55)$. A higher concentration of tCho (mainly due to PCho) was found in hypoxic regions of heterogeneous orthotopic MDA-MB-231 tumor xenografts using three-dimensional multimodal molecular imaging platforms $(56,57)$. The high tCho content of tumor cells was associated with enriched levels of proteins involved in glucose metabolism, PI3K-Akt/Ras/FAS signaling pathway, protein processing in endoplasmic reticulum, apoptosis, and telomere stress-induced senescence (56). A stable silencing of glycerophosphodiester phosphodiesterase domain containing 5 (GDPD5), which is upregulated in TNBC cells and tumors, induced an increase in the levels of GPCho and phosphoethanolamine in MDA-MB-231 BC cells and in their orthotopic tumor xenografts compared with controls $(58,59)$ suggesting a close networking between choline and ethanolamine phospholipid cycles. A larger variation in the PCho/GPCho ratio was observed for the basal-like BC subtype of patient-derived xenograft models relative to luminal B subtype xenografts (60, $61)$. These differences could be explained by lower mRNA expression of ChoK ( $\alpha$ and $\beta$ ) and higher expression of PLA group 4A in basal tumor xenografts (61). In another experimental subcutaneous model expressing basaloid TNBC markers (HCC1806), phosphomonoesters (which include PCho) and lactate levels were modulated by tumor size (62).

The metabolic characterization of TNBC human biopsies is far from being well established, although different studies reported a higher overall tCho content in TBNCs with respect to non-tumoral tissues. A significantly higher tCho level, either quantified in millimoles per kilogram or expressed as peak integral normalized to the volume of interest and the signal-to-noise ratio, was reported in TNBC compared with non-triple-negative tumors using in vivo MRS $(63,64)$. A quantitative study on in vivo MRS examinations of 29 patients indicated that TNBCs exhibited increased tCho levels ranging from 0.4 to $4.9 \mathrm{mmol} / \mathrm{kg}$ (65). 
TNBCs are more likely to have high tCho than other BC subtypes, although the situation may be reversed in young age patients (range 28-39 years) (66). The high tCho levels were well correlated with the standardized FDG uptake value obtained using PET/CT and with the histological prognostic parameters (19). A recent paper (67) reported that intact TNBC biopsies could be discriminated by HR-MAS analyses from triple-positive BCs based on their content in free Cho and GPCho, which were significantly higher in TNBC relative to triple-positive BC. In this paper (67), the analyzed TNBC biopsies contained lower levels of glutamine and higher levels of glutamate compared with tumors with positive receptor statuses which might result from increased glutaminolysis metabolism and suggests dependence on glutamine to support cell growth. This issue warrants measurements of metabolic fluxes using suitable isotopomeric models, such as bonded cumomer analysis by MRS or fragmented cumomer analysis by mass spectrometry $(68,69)$.

\section{TARGETING PtdCho METABOLISM IN TNBC}

The role of PtdCho cycle enzymes as potential new molecular targets in TNBC can be investigated using molecular depletion approaches and/or pharmacological inhibitors. However, there are no studies that allowed discrimination of specific effects of targeting PtdCho-cycle enzymes on TNBC versus other BC subtypes, and most of studies were performed using only one or two TNBC cell lines. Although a reduction of $85 \%$ of in vivo tumor growth was obtained in subcutaneous MDA-MB-231 tumors treated with the ChoK inhibitor Mn58b (70), this result needs further evaluation in different TNBC models. The downregulation of ChoK in MDA-MB-231 cells cultured in vitro induced profound alterations in cell proliferation and promoted differentiation, as detected by cytosolic lipid droplet formation and modified expression of galectin-3 (71). These changes were associated with alterations of 33 proliferation-related genes and 9 DNA repair-related genes (72). Interestingly, in MDA-MB-231cells, a combination of ChoK silencing with a conventional treatment using 5-fluorouracil resulted in higher cell death rate relative to that obtained when each treatment was applied individually (72) confirming a key role for the ChoK enzyme in in vitro cell proliferation and survival. Furthermore, in vivo targeting of ChoK by either lentiviral gene silencing (73) or by ChoK $\alpha$ depletion using specific short-hairpin RNA (shRNA) (74), respectively, induced a growth delay or strongly repressed tumor growth in MDA-MB-231 xenograft bearing mice.

Additionally, there is mounting evidence from studies on experimental TNBC models that the reduction/destabilization of ChoK protein levels rather than inhibition of the activity of this enzyme is more effective in inhibiting tumor growth. In fact, direct or indirect pharmacological inhibitors that were able to reduce the activity of ChoK (and consequently the levels of tCho and PCho) did not reduce cell viability as long as ChoK $\alpha$ protein expression and PtdCho levels were not reduced in TNBC cells grown in vitro (75).
The potential of using PtdCho catabolic pathways as important cotargets for TNBC therapy is gaining relevance. A multitargeting strategy such as simultaneous silencing of PLD1 and ChoK $\alpha$ in MDA-MB-231 cells increased apoptosis (detected by the TUNEL assay) as compared with individual treatments (47). Exposure of MDA-MB-231 cells to D609 which is an inhibitor of a PC-PLC resulted in 60-80\% PC-PLC inhibition associated with tumor cell differentiation, detected by a progressive decrease of mesenchymal traits such as vimentin and N-cadherin expression, reduced galectin-3 and milk fat globule EGF-factor 8 levels, $\beta$-casein formation and decreased in vitro cell migration and invasion (32). These results, obtained from a single tumor model of TNBC, warrant further investigations on a large data set of human TNBCs that can be fully genotyped and metabolically characterized.

\section{FUTURE DIRECTIONS}

Although evidence of specific metabolic alterations in TNBC is accruing, there is a clear need for extending preclinical investigations to a larger number of TNBC models. On the other hand, clinical investigations have to better elucidate the impact of the heterogeneous nature of TNBC lesions on the metabolic profiles and their changes in tumor progression. It may also prove relevant to assess the links between the tCho profile and molecular features such as EGFR overexpression, p53 status, and other specific biological TNBC characteristics. We hypothesize that the PtdCho cycle may represent a good focus point for personalized/precision medicine, offering markers that may be used as diagnosis tools for assessment of cancer prognosis and response to therapy.

The identification of a role for PtdCho metabolism in TNBC progression supports the view that some enzymes of this cycle may act as key regulators of molecular mechanisms leading to cancer onset, invasion, and metastasis, thus representing a new source of potential targets to counteract cancer growth and metastasis.

\section{AUTHOR CONTRIBUTIONS}

The manuscript was written by EI; revised by FP, MJC, RC, SC, FS, GC, and EI; read and approved by all coauthors.

\section{FUNDING}

We acknowledge partial support by Associazione Italiana per la Ricerca sul Cancro (AIRC) 2007-2010; Integrated Oncology Program RO 06.5/N.ISS/Q0, Oncology Program OncOrd 37/07/N. ISS/70CF/4 and Special Program Alleanza Contro il Cancro 2006, ACC3-AC5/D, Ministry of Health, Italy; Accordo di Collaborazione Italia-USA ISS/530F/0F29; AIRC 20092011, IG N. 9147, and AIRC IG 129762012 and Programma Oncotecnologico ISS/13ONC/5. MJC was funded by TRAIN programme, Italy (Cofunded by Marie Curie Actions, 7th Framework Programme), Incoming Grant no. 246549. 


\section{REFERENCES}

1. Perou CM, Sorlie T, Eisen MB, van de Rijn M, Jeffrey SS, Rees CA, et al. Molecular portraits of human breast tumours. Nature (2000) 406(6797):747-52. doi:10.1038/35021093

2. Sorlie T, Tibshirani R, Parker J, Hastie T, Marron JS, Nobel A, et al. Repeated observation of breast tumor subtypes in independent gene expression data sets. ProcNatlAcadSciUSA (2003) 100(14):8418-23. doi:10.1073/pnas.0932692100

3. Prat A, Adamo B, Cheang MC, Anders CK, Carey LA, Perou CM. Molecular characterization of basal-like and non-basal-like triple-negative breast cancer. Oncologist (2013) 18(2):123-33. doi:10.1634/theoncologist.2012-0397

4. Foulkes WD, Smith IE, Reis-Filho JS. Triple-negative breast cancer. N Engl J Med (2010) 363(20):1938-48. doi:10.1056/NEJMra1001389

5. Podo F, Buydens LM, Degani H, Hilhorst R, Klipp E, Gribbestad IS, et al. Triple-negative breast cancer: present challenges and new perspectives. $\mathrm{Mol}$ Oncol (2010) 4(3):209-29. doi:10.1016/j.molonc.2010.04.006

6. Burstein MD, Tsimelzon A, Poage GM, Covington KR, Contreras A, Fuqua SA, et al. Comprehensive genomic analysis identifies novel subtypes and targets of triple-negative breast cancer. Clin Cancer Res (2015) 21(7):1688-98. doi:10.1158/1078-0432.CCR-14-0432

7. Price JT, Tiganis T, Agarwal A, Djakiew D, Thompson EW. Epidermal growth factor promotes MDA-MB-231 breast cancer cell migration through a phosphatidylinositol 3'-kinase and phospholipase C-dependent mechanism. Cancer Res (1999) 59(21):5475-8.

8. Corkery B, Crown J, Clynes M, O’Donovan N. Epidermal growth factor receptor as a potential therapeutic target in triple-negative breast cancer. Ann Oncol (2009) 20(5):862-7. doi:10.1093/annonc/mdn710

9. Podo F, Canevari S, Canese R, Pisanu ME, Ricci A, Iorio E. MR evaluation of response to targeted treatment in cancer cells. NMR Biomed (2011) 24(6):648-72. doi:10.1002/nbm.1658

10. Tyanova S, Albrechtsen R, Kronqvist P, Cox J, Mann M, Geiger T. Proteomic maps of breast cancer subtypes. Nat Commun (2016) 7:10259. doi:10.1038/ ncomms 10259

11. Xu H, Eirew P, Mullaly SC, Aparicio S. The omics of triple-negative breast cancers. Clin Chem (2014) 60(1):122-33. doi:10.1373/clinchem.2013.207167

12. Choi J, Kim do H, Jung WH, Koo JS. Metabolic interaction between cancer cells and stromal cells according to breast cancer molecular subtype. Breast Cancer Res (2013) 15(5):R78. doi:10.1186/bcr3472

13. Kanaan YM, Sampey BP, Beyene D, Esnakula AK, Naab TJ, Ricks-Santi LJ, et al. Metabolic profile of triple-negative breast cancer in African-American women reveals potential biomarkers of aggressive disease. Cancer Genomics Proteomics (2014) 11(6):279-94.

14. Pinheiro C, Sousa B, Albergaria A, Paredes J, Dufloth R, Vieira D, et al. GLUT1 and CAIX expression profiles in breast cancer correlate with adverse prognostic factors and MCT1 overexpression. Histol Histopathol (2011) 26(10):1279-86.

15. Shen L, O'Shea JM, Kaadige MR, Cunha S, Wilde BR, Cohen AL, et al. Metabolic reprogramming in triple-negative breast cancer through Myc suppression of TXNIP. Proc Natl Acad Sci U S A (2015) 112(17):5425-30. doi:10.1073/pnas.1501555112

16. Lim SO, Li CW, Xia W, Lee HH, Chang SS, Shen J, et al. EGFR signaling enhances aerobic glycolysis in triple-negative breast cancer cells to promote tumor growth and immune escape. Cancer Res (2016) 76(5):1284-96. doi:10.1158/0008-5472.CAN-15-2478

17. Pelicano H, Zhang W, Liu J, Hammoudi N, Dai J, Xu RH, et al. Mitochondrial dysfunction in some triple-negative breast cancer cell lines: role of mTOR pathway and therapeutic potential. Breast Cancer Res (2014) 16(5):434. doi:10.1186/s13058-014-0434-6

18. Chiacchio S, Evangelista L, AlSharif A, Manca G, Di Martino F, Negri A, et al. Association between semiquantitative Pet parameters and molecular subtypes of breast invasive ductal carcinoma. Q J Nucl Med Mol Imaging (2015).

19. Koo HR, Park JS, Kang KW, Cho N, Chang JM, Bae MS, et al. 18F-FDG uptake in breast cancer correlates with immunohistochemically defined subtypes. Eur Radiol (2014) 24(3):610-8. doi:10.1007/s00330-013-3037-1

20. Tozaki M, Hoshi K. $1 \mathrm{H}$ MR spectroscopy of invasive ductal carcinoma: correlations with FDG PET and histologic prognostic factors. AJR Am J Roentgenol (2010) 194(5):1384-90. doi:10.2214/AJR.09.3431

21. Yue Y, Cui X, Bose S, Audeh W, Zhang X, Fraass B. Stratifying triple-negative breast cancer prognosis using 18F-FDG-PET/CT imaging. Breast Cancer Res Treat (2015) 153(3):607-16. doi:10.1007/s10549-015-3558-1
22. Hilvo M, Denkert C, Lehtinen L, Muller B, Brockmoller S, Seppanen-Laakso $\mathrm{T}$, et al. Novel theranostic opportunities offered by characterization of altered membrane lipid metabolism in breast cancer progression. Cancer Res (2011) 71(9):3236-45. doi:10.1158/0008-5472.CAN-10-3894

23. Menendez JA, Lupu R. Fatty acid synthase and the lipogenic phenotype in cancer pathogenesis. Nat Rev Cancer (2007) 7(10):763-77. doi:10.1038/nrc2222

24. Currie E, Schulze A, Zechner R, Walther TC, Farese RV Jr. Cellular fatty acid metabolism and cancer. Cell Metab (2013) 18(2):153-61. doi:10.1016/ j.cmet.2013.05.017

25. Flores FH, Viñas G, Oliveras G, Giro-Perafita A, Perez-Bueno F, Roque A, et al. Triple negative breast cancer: clinicopathologic characteristics and fatty acid synthase (FASN) expression as a potential target. Ann Oncol (2014) 25(4 Suppl):iv58-84. doi:10.1093/annonc/mdu326.6

26. Viñas G, Oliveras G, Perez-Bueno F, Giro A, Blancafort A, Puig-Vives M, et al. Fatty acid synthase (FASN) expression in triple-negative breast cancer. Cancer Res (2012) 72(24 Suppl):P4-09-11. doi:10.1158/0008-5472.sabcs12-p4-09-11

27. Kim S, Lee Y, Koo JS. Differential expression of lipid metabolism-related proteins in different breast cancer subtypes. PLoS One (2015) 10(3):e0119473. doi:10.1371/journal.pone.0119473

28. Thapa N, Choi S, Tan X, Wise T, Anderson RA. Phosphatidylinositol phosphate 5-kinase igamma and phosphoinositide 3-kinase/Akt signaling couple to promote oncogenic growth. J Biol Chem (2015) 290(30):18843-54. doi:10.1074/jbc.M114.596742

29. Pisterzi P, Mercurio L, Caramujo MJ, Iorio E, Podo F, Cecchetti S. Phosphatidylcholine-specific phospholipase $C$ inhibition as a new therapeutic approach to control triple-negative breast cancer cells proliferation. Proceedings of the Special Conference EACR-AACR-SIC. Florence (2015).

30. Paris L, Cecchetti S, Spadaro F, Abalsamo L, Lugini L, Pisanu ME, et al. Inhibition of phosphatidylcholine-specific phospholipase $\mathrm{C}$ downregulates HER2 overexpression on plasma membrane of breast cancer cells. Breast Cancer Res (2010) 12(3):R27. doi:10.1186/bcr2575

31. Miyake T, Parsons SJ. Functional interactions between choline kinase alpha, epidermal growth factor receptor and c-Src in breast cancer cell proliferation. Oncogene (2012) 31(11):1431-41. doi:10.1038/onc.2011.332

32. Abalsamo L, Spadaro F, Bozzuto G, Paris L, Cecchetti S, Lugini L, et al. Inhibition of phosphatidylcholine-specific phospholipase $\mathrm{C}$ results in loss of mesenchymal traits in metastatic breast cancer cells. Breast Cancer Res (2012) 14(2):R50. doi:10.1186/bcr3151

33. Podo F, Paris L, Cecchetti S, Spadaro F, Abalsamo L, Ramoni C, et al. Activation of phosphatidylcholine-specific phospholipase $\mathrm{C}$ in breast and ovarian cancer: impact on MRS-detected choline metabolic profile and perspectives for targeted therapy. Front Oncol (2016) 6:171. doi:10.3389/fonc.2016.00171

34. Beloueche-Babari M, Chung YL, Al-Saffar NM, Falck-Miniotis M, Leach MO. Metabolic assessment of the action of targeted cancer therapeutics using magnetic resonance spectroscopy. Br J Cancer (2010) 102(1):1-7. doi:10.1038/ sj.bjc. 6605457

35. Glunde K, Bhujwalla ZM, Ronen SM. Choline metabolism in malignant transformation. Nat Rev Cancer (2011) 11(12):835-48. doi:10.1038/nrc3162

36. Podo F. Tumour phospholipid metabolism. NMR Biomed (1999) 12(7):413-39. doi:10.1002/(SICI)1099-1492(199911)12:7<413::AID-NBM587>3.0.CO;2-U

37. Sardanelli F, Fausto A, Di Leo G, de Nijs R, Vorbuchner M, Podo F. In vivo proton MR spectroscopy of the breast using the total choline peak integral as a marker of malignancy. AJR Am J Roentgenol (2009) 192(6):1608-17. doi:10.2214/AJR.07.3521

38. Aboagye EO, Bhujwalla ZM. Malignant transformation alters membrane choline phospholipid metabolism of human mammary epithelial cells. Cancer Res (1999) 59(1):80-4.

39. Eliyahu G, Kreizman T, Degani H. Phosphocholine as a biomarker of breast cancer: molecular and biochemical studies. Int J Cancer (2007) 120(8):1721-30. doi:10.1002/ijc. 22293

40. Glunde K, Jie C, Bhujwalla ZM. Molecular causes of the aberrant choline phospholipid metabolism in breast cancer. Cancer Res (2004) 64(12):4270-6. doi:10.1158/0008-5472.CAN-03-3829

41. Ackerstaff E, Pflug BR, Nelson JB, Bhujwalla ZM. Detection of increased choline compounds with proton nuclear magnetic resonance spectroscopy subsequent to malignant transformation of human prostatic epithelial cells. Cancer Res (2001) 61(9):3599-603.

42. Canese R, Pisanu ME, Mezzanzanica D, Ricci A, Paris L, Bagnoli M, et al. Characterisation of in vivo ovarian cancer models by quantitative $1 \mathrm{H}$ 
magnetic resonance spectroscopy and diffusion-weighted imaging. NMR Biomed (2012) 25(4):632-42. doi:10.1002/nbm.1779

43. Iorio E, Mezzanzanica D, Alberti P, Spadaro F, Ramoni C, D’Ascenzo S, et al. Alterations of choline phospholipid metabolism in ovarian tumor progression. Cancer Res (2005) 65(20):9369-76. doi:10.1158/0008-5472.CAN-05-1146

44. Iorio E, Ricci A, Bagnoli M, Pisanu ME, Castellano G, Di Vito M, et al. Activation of phosphatidylcholine cycle enzymes in human epithelial ovarian cancer cells. Cancer Res (2010) 70(5):2126-35. doi:10.1158/0008-5472. CAN-09-3833

45. Fagone P, Jackowski S. Phosphatidylcholine and the CDP-choline cycle. Biochim Biophys Acta (2013) 1831(3):523-32. doi:10.1016/j.bbalip.2012.09.009

46. Vance JE, Vance DE. Phospholipid biosynthesis in mammalian cells. Biochem Cell Biol (2004) 82(1):113-28. doi:10.1139/003-073

47. Gadiya M, Mori N, Cao MD, Mironchik Y, Kakkad S, Gribbestad IS, et al. Phospholipase D1 and choline kinase-alpha are interactive targets in breast cancer. Cancer Biol Ther (2014) 15(5):593-601. doi:10.4161/cbt.28165

48. Glunde K, Mori N, Takagi T, Cecchetti S, Ramoni C, Iorio E, et al. Choline kinase silencing in breast cancer cells results in compensatory upregulation of phosphatidylcholine-specific phospholipase C. Proc Int Soc Mag Reson Med (2008) 16:244.

49. Podo F, Glunde K, Cecchetti S, Krishnamachary B, Mori N, Paris L, et al. Cross-talk between enzymes responsible for the elevated 1H MRS phosphocholine signal in breast cancer cells. Proc of the Sixth Annual World Molecular Imaging Congress (WMIC). Savannah, GA (2013).

50. Brglez V, Pucer A, Pungercar J, Lambeau G, Petan T. Secreted phospholipases A(2)are differentially expressed and epigenetically silenced in human breast cancer cells. Biochem Biophys Res Commun (2014) 445(1):230-5. doi:10.1016/ j.bbrc.2014.01.182

51. Beckmann MW, Niederacher D, Schnurch HG, Gusterson BA, Bender HG. Multistep carcinogenesis of breast cancer and tumour heterogeneity. J Mol Med (Berl) (1997) 75(6):429-39. doi:10.1007/s001090050128

52. Caramujo MJ, Cecchetti S, Pisterzi P, de Carvalho CCCR, Podo F, Iorio E. Metabolite assessment of breast cancer cells offers evidence of altered phospholipid metabolism. Magn Reson Mater Phy (2015) 28(S1):S458. doi:10.1007/s10334-015-0490-7

53. He M, Guo S, Li Z. In situ characterizing membrane lipid phenotype of breast cancer cells using mass spectrometry profiling. Sci Rep (2015) 5:11298. doi:10.1038/srep11298

54. Mori N, Glunde K, Takagi T, Bhujwalla ZM. The choline phospholipid metabolism detected by comparing cancer cells with tumors. Proc Int Soc Magn Reson Med (2008) 16:2800.

55. Mori N, Wildes F, Takagi T, Glunde K, Bhujwalla ZM. Differences in phospholipid and lipid metabolism between cancer cells in culture and in solid tumors. Proc Int Soc Magn Reson Med (2015) 23:1126.

56. Jiang L, Chughtai K, Purvine SO, Bhujwalla ZM, Raman V, Pasa-Tolic L, et al. MALDI-mass spectrometric imaging revealing hypoxia-driven lipids and proteins in a breast tumor model. Anal Chem (2015) 87(12):5947-56. doi:10.1021/ac504503x

57. Jiang L, Greenwood TR, Artemov D, Raman V, Winnard PT Jr, Heeren RM, et al. Localized hypoxia results in spatially heterogeneous metabolic signatures in breast tumor models. Neoplasia (2012) 14(8):732-41. doi:10.1593/ neo. 12858

58. Cao MD, Dopkens M, Krishnamachary B, Vesuna F, Gadiya MM, Lonning PE, et al. Glycerophosphodiester phosphodiesterase domain containing 5 (GDPD5) expression correlates with malignant choline phospholipid metabolite profiles in human breast cancer. NMR Biomed (2012) 25(9):1033-42. doi: $10.1002 / \mathrm{nbm} .2766$

59. Wijnen JP, Jiang L, Greenwood TR, Cheng M, Dopkens M, Cao MD, et al. Silencing of the glycerophosphocholine phosphodiesterase GDPD5 alters the phospholipid metabolite profile in a breast cancer model in vivo as monitored by (31) P MRS. NMR Biomed (2014) 27(6):692-9. doi:10.1002/ nbm.3106

60. Grinde MT, Skrbo N, Moestue SA, Rodland EA, Borgan E, Kristian A, et al. Interplay of choline metabolites and genes in patient-derived breast cancer xenografts. Breast Cancer Res (2014) 16(1):R5. doi:10.1186/bcr3597

61. Moestue SA, Borgan E, Huuse EM, Lindholm EM, Sitter B, Borresen-Dale AL, et al. Distinct choline metabolic profiles are associated with differences in gene expression for basal-like and luminal-like breast cancer xenograft models. BMC Cancer (2010) 10:433. doi:10.1186/1471-2407-10-433
62. Liu T, Nath K, Liu W, Zhou R, Chen IW. A study of the relationship of metabolic MR parameters to estrogen dependence in breast cancer xenografts. NMR Biomed (2015) 28(9):1087-96. doi:10.1002/nbm.3342

63. Chen JH, Mehta RS, Baek HM, Nie K, Liu H, Lin MQ, et al. Clinical characteristics and biomarkers of breast cancer associated with choline concentration measured by 1H MRS. NMR Biomed (2011) 24(3):316-24. doi:10.1002/ nbm. 1595

64. Shin HJ, Baek HM, Cha JH, Kim HH. Evaluation of breast cancer using proton MR spectroscopy: total choline peak integral and signal-to-noise ratio as prognostic indicators. AJR Am J Roentgenol (2012) 198(5):W488-97. doi:10.2214/ AJR.11.7292

65. Chen JH, Agrawal G, Feig B, Baek HM, Carpenter PM, Mehta RS, et al. Triple-negative breast cancer: MRI features in 29 patients. Ann Oncol (2007) 18(12):2042-3. doi:10.1093/annonc/mdm504

66. Sah RG, Sharma U, Parshad R, Seenu V, Mathur SR, Jagannathan NR. Association of estrogen receptor, progesterone receptor, and human epidermal growth factor receptor 2 status with total choline concentration and tumor volume in breast cancer patients: an MRI and in vivo proton MRS study. Magn Reson Med (2012) 68(4):1039-47. doi:10.1002/mrm.24117

67. Cao MD, Lamichhane S, Lundgren S, Bofin A, Fjosne H, Giskeodegard GF, et al. Metabolic characterization of triple negative breast cancer. BMC Cancer (2014) 14:941. doi:10.1186/1471-2407-14-941

68. Shestov AA, Mancuso A, Lee SC, Guo L, Nelson DS, Roman JC, et al. Bonded cumomer analysis of human melanoma metabolism monitored by 13C NMR spectroscopy of perfused tumor cells. J Biol Chem (2016) 291(10):5157-71. doi:10.1074/jbc.M115.701862

69. Shestov AA, Lee SC, Nath K, Guo L, Nelson DS, Roman JC, et al. (13)C MRS and LC-MS flux analysis of tumor intermediary metabolism. Front Oncol (2016) 6:135. doi:10.3389/fonc.2016.00135

70. Ramirez de Molina A, Banez-Coronel M, Gutierrez R, Rodriguez-Gonzalez A, Olmeda D, Megias D, et al. Choline kinase activation is a critical requirement for the proliferation of primary human mammary epithelial cells and breast tumor progression. Cancer Res (2004) 64(18):6732-9. doi:10.1158/0008-5472. CAN-04-0489

71. Glunde K, Raman V, Mori N, Bhujwalla ZM. RNA interference-mediated choline kinase suppression in breast cancer cells induces differentiation and reduces proliferation. Cancer Res (2005) 65(23):11034-43. doi:10.1158/ 0008-5472.CAN-05-1807

72. Mori N, Glunde K, Takagi T, Raman V, Bhujwalla ZM. Choline kinase down-regulation increases the effect of 5-fluorouracil in breast cancer cells. Cancer Res (2007) 67(23):11284-90. doi:10.1158/0008-5472.CAN-07-2728

73. Krishnamachary B, Glunde K, Wildes F, Mori N, Takagi T, Raman V, et al. Noninvasive detection of lentiviral-mediated choline kinase targeting in a human breast cancer xenograft. Cancer Res (2009) 69(8):3464-71. doi:10.1158/0008-5472.CAN-08-4120

74. Mignion L, Danhier P, Magat J, Porporato PE, Masquelier J, Gregoire V, et al. Non-invasive in vivo imaging of early metabolic tumor response to therapies targeting choline metabolism. Int J Cancer (2016) 138(8):2043-9. doi:10.1002/ ijc. 29932

75. Mori N, Wildes F, Kakkad S, Jacob D, Solaiyappan M, Glunde K, et al. Choline kinase-alpha protein and phosphatidylcholine but not phosphocholine are required for breast cancer cell survival. NMR Biomed (2015) 28(12):1697-706. doi:10.1002/nbm.3429

Conflict of Interest Statement: The authors declare that the research was conducted in the absence of any commercial or financial relationships that could be construed as a potential conflict of interest.

The reviewer BK and handling editor declared their shared affiliation, and the handling editor states that the process nevertheless met the standards of a fair and objective review.

Copyright $\odot 2016$ Iorio, Caramujo, Cecchetti, Spadaro, Carpinelli, Canese and Podo. This is an open-access article distributed under the terms of the Creative Commons Attribution License (CC BY). The use, distribution or reproduction in other forums is permitted, provided the original author(s) or licensor are credited and that the original publication in this journal is cited, in accordance with accepted academic practice. No use, distribution or reproduction is permitted which does not comply with these terms. 